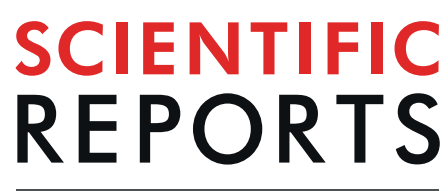

natureresearch

\title{
Towards standardisation of contact and contactless electrical measurements of CVD graphene at the macro-, micro- and nano-scale
}

\author{
Christos Melios ${ }^{1,2}{ }^{2}$ Nathaniel Huang ${ }^{1}$, Luca Callegaro ${ }^{3}$, Alba Centeno ${ }^{4}$, Alessandro Cultrera ${ }^{3}$, \\ Alvaro Cordon ${ }^{5}$, Vishal Panchal ${ }^{1}$, Israel Arnedo ${ }^{5}$, Albert Redo-Sanchez ${ }^{5}$, David Etayo ${ }^{5}$, \\ Montserrat Fernandez ${ }^{5}$, Alex Lopez ${ }^{5}$, Sergiy Rozhko ${ }^{1}$, Oihana Txoperena ${ }^{4}$, Amaia Zurutuza ${ }^{4}$ \& \\ Olga Kazakova ${ }^{1^{*}}$
}

Graphene has become the focus of extensive research efforts and it can now be produced in waferscale. For the development of next generation graphene-based electronic components, electrical characterization of graphene is imperative and requires the measurement of work function, sheet resistance, carrier concentration and mobility in both macro-, micro- and nano-scale. Moreover, commercial applications of graphene require fast and large-area mapping of electrical properties, rather than obtaining a single point value, which should be ideally achieved by a contactless measurement technique. We demonstrate a comprehensive methodology for measurements of the electrical properties of graphene that ranges from nano- to macro-scales, while balancing the acquisition time and maintaining the robust quality control and reproducibility between contact and contactless methods. The electrical characterisation is achieved by using a combination of techniques, including magneto-transport in the van der Pauw geometry, THz time-domain spectroscopy mapping and calibrated Kelvin probe force microscopy. The results exhibit excellent agreement between the different techniques. Moreover, we highlight the need for standardized electrical measurements in highly controlled environmental conditions and the application of appropriate weighting functions.

The unique properties of graphene ${ }^{1}$ and the increasing demand for large-scale production were the reason for the development of various industrial methods to grow this material. One of the most promising methods is the use of CVD to grow graphene onto various metallic substrates. In CVD growth, graphene is grown on the surface of the metal after hydrocarbons decompose ${ }^{2}$. The most promising metal substrate used until now is $\mathrm{Cu}^{3}$, however graphene is currently grown also on $\mathrm{Ni}(111)^{4-6}, \mathrm{Ru}(0001)^{7}, \mathrm{Pt}(111)^{8,9}$, and $\operatorname{Ir}(111)^{9-11}$. Subsequently to the growth process, for graphene to be use in electronic applications, it needs to be transferred on an insulating substrate (i.e. $\mathrm{Si} / \mathrm{SiO}_{2}$, quartz or polyethylene terephthalate (PET), which is a transparent and flexible substrate). The already happening now use of graphene in RF electronics ${ }^{12}$, integrated circuits ${ }^{13}$ and optoelectronics ${ }^{14}$ has triggered impressive progress in large-scale production, however the community still lacks standardised electrical measurements to extract useful parameters such as carrier concentration, mobility and sheet resistance, all of those are often presented as figures-of-merit of the graphene quality. Currently, the most widely used method for electrical characterisation involves magneto-transport measurements in lithographically patterned Hall bars to extract carrier concentration, mobility and resistance. However, this method is not suitable for high throughput characterisation and often the measured graphene is significantly altered due to the microfabrication processes, in which case the electrical properties of the pristine transferred graphene are different from the ones measured. An alternative method for mapping the sheet resistance of graphene films is using micro four-point probes (M4PP) $)^{15,16}$. Recently the use of microwave cavities ${ }^{16-18}$ and Terahertz time-domain

\footnotetext{
${ }^{1}$ National Physical Laboratory, Teddington, TW11 0LW, United Kingdom. ${ }^{2}$ Department of Electrical and Computer Engineering, Faculty of Engineering, University of Cyprus, 1687, Nicosia, Cyprus. ${ }^{3}$ Istituto Nazionale di Ricerca Metrologica, Strada delle Cacce 91, 10135, Torino, Italy. ${ }^{4}$ Graphenea SA, 20018, Donostia-San Sebastián, Spain. ${ }^{5}$ DasNano, Poligono Industrial Talluntxe II, Calle M-10, 31192, Tajonar, Navarra, Spain. *email: olga.kazakova@npl.co.uk
} 
Spectroscopy (THz-TDS $)^{16,19-23}$ measurements provided a non-contact method of measuring the conductivity of graphene films. Despite the attractiveness of these techniques, fluctuations in ambient conditions (such as humidity) affect the electrical properties of graphene ${ }^{24,25}$, resulting in discriminations between measurements at different laboratories.

In contrast to the conventional Hall effect, where device fabrication is required for carrier concentration and mobility measurements, the van der Pauw (vdP) method only requires thin uniform samples with relatively small Ohmic contacts ${ }^{26}$. This method was originally introduced by van der Pauw in 1958 to measure the resistivity and Hall coefficient of arbitrary shape semiconductors ${ }^{27,28}$.

$\mathrm{THz}$-TDS has recently been demonstrated to be an excellent tool to characterize the quality and conductivity of graphene deposited on large areas with suitable choices of substrates and models. THz-TDS can provide a map of the absolute value of conductivity/resistivity in a non-contact and non-destructive manner. The measurement is fast and can cover the entire area of a sample and can be used in all stages of a material development since the prototyping up to manufacturing scale ${ }^{29-31}$.

An important property of graphene is its work function $\left(\Phi_{G r}\right)$. Currently, photoemission electron microscopy $(\mathrm{PEEM})^{32}$ is commonly used for measuring the local work function and distribution of graphene layers with nanometre resolution. Nevertheless, this technique requires ultra-high vacuum to operate and the surface must be completely clean, meaning that high temperature annealing $\left(\sim 400^{\circ} \mathrm{C}\right)$ is used prior the measurement. Both conditions pose a significant drawback when rapid characterisation is needed. An alternative, complementary technique that can provide valuable information about local electrical properties, such as surface potential, doping and work function as well as providing essential information about layer distribution down to $\sim 20 \mathrm{~nm}$ spatial resolution is frequency-modulated Kelvin probe force microscopy (FM-KPFM) ${ }^{33,34}$. KPFM has already demonstrated great potential for graphene characterisation, identification of graphene layers as well as measuring the surface potential of the samples with nanometre resolution ${ }^{24,35-40}$.

In this paper, we propose and demonstrate a standardised procedure for measuring and mapping the electrical properties of scalable graphene using a combination of contact and contactless complementary methods, such as van der Pauw magneto-transport, THz-TDS conductivity mapping and Kelvin probe force microscopy. The methodologies here described are currently being developed within the GRACE project ${ }^{41}$. They will become part of Good Practice Guides for the measurement of electrical properties of graphene and will be proposed as an input to normative bodies, towards the development of documentary standards. In this work, we demonstrate appropriate measurement techniques to characterise the most important electrical properties of graphene from macroscale down to nanoscale and establish the validity of the measurements through correlation in an inter-laboratory study and applying a weighting correction function between the results obtained from THz-TDS and van der Pauw methods.

\section{Measurements of Electrical Properties on Different Scales}

Confocal laser scanning microscopy. Confocal laser scanning microscopy (CLSM) is a powerful optical technique that enables rapid structural characterisation of large-area graphene and graphene nanostructures by pushing the spatial resolution beyond the optical diffraction limit and stacking individual images of different focal planes. CLSM was performed using an Olympus LEXT OLS4100 system equipped with $5 \times, 10 \times, 20 \times, 50 \times$ and $100 \times$ objectives (numerical apertures: $0.15,0.30,0.60,0.95$ and 0.95 , respectively) and with further $1 \times$ to $8 \times$ optical zoom. Therefore, images of a field-of-view ranging from $2560 \mu \mathrm{m}$ to $16 \mu \mathrm{m}$ can be captured. The system uses a $405 \mathrm{~nm}$ wavelength laser, which scans in X-Y directions using an electromagnetic micro-electromechanical system (MEMS) scanner and a high-precision galvano-mirror. The optical images (of up to $4096 \times 4096$ pixels with horizontal spatial resolution down to $120 \mathrm{~nm}$ ) are generated using a photomultiplier which captures the reflected light. In this confocal setup, in-focus reflected light is only allowed to pass through the circular confocal pinhole, therefore eliminating flare from out-of-focus areas. Due to the shallow depth-of-field of the CLSM setup, the objective is moved vertically to capture images of different focal planes. Using this Z-stacking technique, a single output of the $2 \mathrm{D}$ reflected intensity map is extracted by determining the maximum brightness value of the calculated Intensity- $Z$ curves for each pixel from the stack of images. The system is operated in ambient air and does not require any sample preparation. For these measurements, individual images with high magnification $(100 \times$ objective $)$ were captured and then stitched together to generate a high resolution image of a larger area ${ }^{42}$.

Kelvin probe force microscopy. FM-KPFM uses the force gradient $\left(\frac{d F}{d z}\right)$ to calculate surface potential $\left(U_{C P D}\right)$. This is done by measuring a mechanical resonant frequency shift of a cantilever either in single or double pass. In single-pass FM-KPFM, the cantilever is oscillating at its mechanical resonant frequency $f_{0} \approx 300 \mathrm{kHz}$, with an AC voltage of much lower frequency $f_{\text {mod }} \approx 3 \mathrm{kHz}$ of $5 \mathrm{~V}$ amplitude also applied, to induce a frequency shift of $f_{0} \pm f_{\text {mod }}$. The side lobes (monitored by a PID feedback loop) generated by this shift are minimized by applying a DC compensation voltage. By measuring this DC voltage at each pixel, a surface potential map [i.e. contact potential difference $\left.\left(U_{C P D}\right)\right]$ is constructed ${ }^{33,35,36}$. The measurements were performed in ambient conditions $\left(32 \% \mathrm{RH}, 22^{\circ} \mathrm{C}\right)$. Since FM-KPFM measures the surface potential (difference in work function between the tip and the sample), it provides a qualitative result, highly dependent on the tip status. In our approach we demonstrate a method to appropriately calibrate the work function of the scanning tip $\left(\Phi_{T i p}\right)$ to quantitative measure the absolute work function of the graphene samples. The calibration method is presented in Fig. 1. Firstly, freshly cleaved highly oriented pyrolytic graphite (HOPG) is measured using ultra-violet photoelectron spectroscopy (UPS). Therefore the work function of HOPG is calculated using $\Phi_{H O P G}=h v-x=4.48 \mathrm{eV}$, where $h v=21.22 \mathrm{eV}$ is the photon energy and $x$ is the energy difference between the Fermi edge and cut-off (for more experimental details on UPS see ref. ${ }^{24}$ ). Then the same HOPG reference sample is measured using FM-KPFM, and the tip is calibrated using $\Phi_{T i p}=\Phi_{H O P G}+e U_{C P D}^{H O P G}$, where $U_{C P D}^{H O P G}$ is the surface potential measured using 

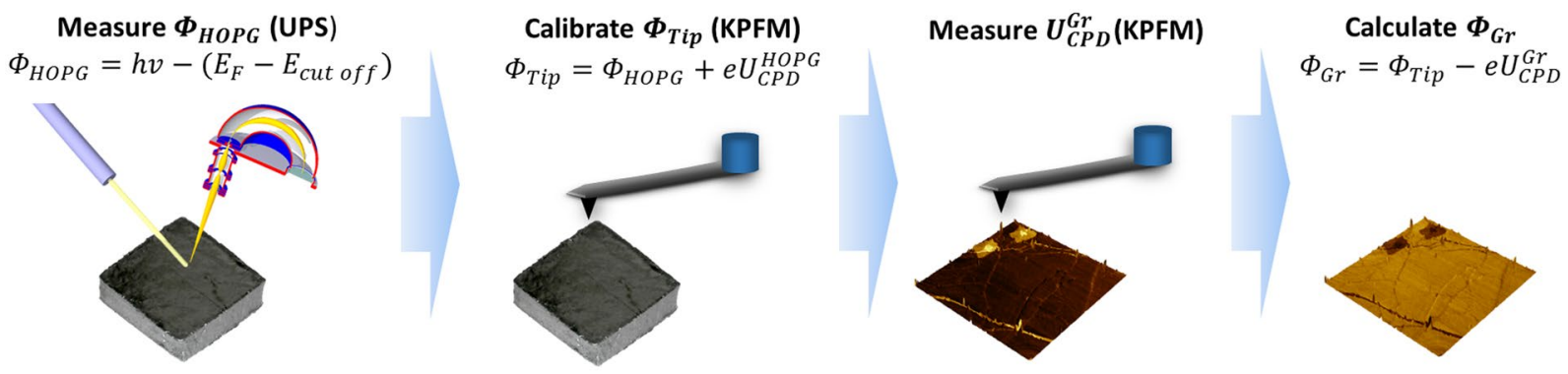

Figure 1. Measurement and calibration procedure for definition of local work function in graphene samples. The dark rectangles in the upmost right image (work function map) correspond to bilayer graphene with lowest values of work function.

FM-KPFM. Subsequently to the calibration, the graphene sample is measured, and the work function can be calculated using $\Phi_{G r}=\Phi_{T i p}-e U_{C P D}^{G r}$. A good practice is to check the calibration of the tip after any measurements to ensure no drift or error.

THz-TDS sheet resistance mapping. The extraction of the resistivity of a thin film on a substrate using THz-TDS in reflection geometry $\left(R_{s}^{T H z}\right)$ is based on measuring the difference of the THz reflectivity of the film on top of the substrate and the reflectivity of the bare substrate. In transmission geometry, the conductivity is extracted by comparing the signal of the sample with the thin film and the signal of the bare substrate in transmission mode.

The reflectivity is governed by the difference in refraction index of the film and substrate and the Fresnel equations. The relative amplitude of these two $\mathrm{THz}$ time-domain reflectivities analysed in the frequency-domain via a Fourier transform of each individual pulse allows estimating the contribution in the resistivity of the thin film on top of the substrate.

The THz-TDS measurement where carried out with das-Nano Onyx 2D materials quality-inspector system. This system generates maps of sheet resistance of the entire area of a sample in a non-destructive and non-contact way. The measurements are realized in normal reflection. The maps were acquired on an area of $10 \times 10 \mathrm{~mm}$ with a step size of $100 \mu \mathrm{m}$ and the acquisition took about 5 minutes. The maps were generated at $0.5 \mathrm{THz}$. The measurements were conducted at room temperature between $24-26^{\circ} \mathrm{C}$ and relative humidity between $35-50 \%$.

Magneto-transport measurements in the van der Pauw geometry. VdP measurements are performed by placing contacts at the edges (periphery) of the sample, passing current and measuring voltages in different configurations ${ }^{27,28}$. The samples used in this work have square shape, therefore contacts are placed at the corners (see Fig. 2). The vdP method can be described by two modes of operation: (i) sheet resistance $\left(R_{s}^{v d P}\right)$ and (ii) Hall measurements $\left(R_{H}\right)$.

Sheet resistance measurements. By applying current and measuring the voltage drop at the opposite sides and repeating the operation by rotating the sample by $90^{\circ}$ (Fig. 2a), 8 resistance values can be calculated:

$$
\begin{aligned}
& R_{43,12}=\frac{V_{43}}{I_{12}} \quad R_{34,21}=\frac{V_{34}}{I_{21}} \\
& R_{32,41}=\frac{V_{32}}{I_{41}} \quad R_{23,14}=\frac{V_{23}}{I_{14}} \\
& R_{12,43}=\frac{V_{12}}{I_{43}} \quad R_{21,34}=\frac{V_{21}}{I_{34}} \\
& R_{41,32}=\frac{V_{41}}{I_{32}} \quad R_{14,23}=\frac{V_{14}}{I_{23}}
\end{aligned}
$$

Using the values from Eq. (1), the resistances $R_{A}$ (current $I_{14}, I_{32}$ ) and $R_{B}$ (current $I_{21}, I_{43}$ ) can be derived:

$$
R_{A}=\frac{R_{43,12}+R_{34,21}+R_{12,43}+R_{21,34}}{4}
$$

and

$$
R_{B}=\frac{R_{32,41}+R_{23,14}+R_{41,32}+R_{14,23}}{4}
$$

Therefore, the sheet resistance $R_{s}^{v d P}$ of the graphene sample is calculated numerically using: 
(a) Sheet resistance $\left(R_{S}\right)$ measurements

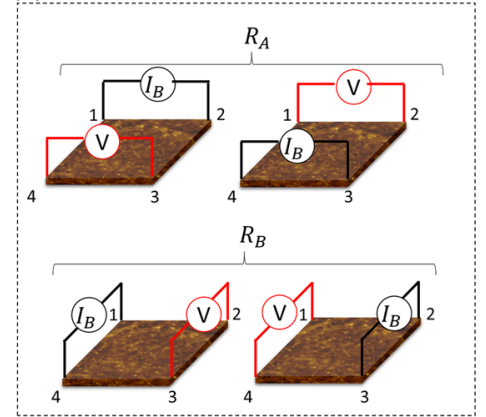

(b)

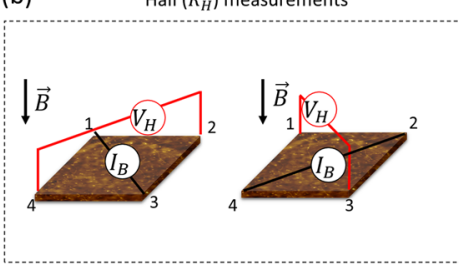

(c)

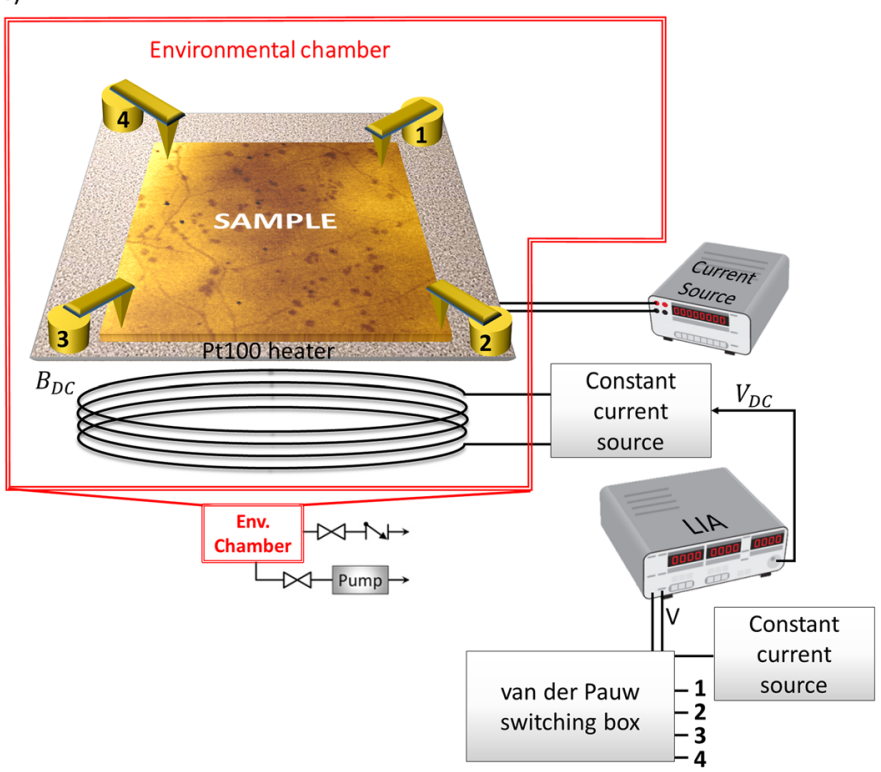

Figure 2. Schematics of van der Pauw configurations for (a) sheet resistance and (b) Hall effect measurements. (c) Experimental setup of magneto-transport measurements of un-patterned samples in the vdP geometry using AC bias and DC magnetic field. In all the cases, the sample in the schematics is represented by an experimental KPFM map of a CVD graphene sample.

$$
\exp \left(\frac{-\pi R_{A}}{R_{s}^{v d P}}\right)+\exp \left(\frac{-\pi R_{B}}{R_{s}^{v d P}}\right)=1
$$

Hall coefficient measurements. For the Hall coefficient measurements, the sample is placed in a perpendicular magnetic field $(B)$. A bias current $\left(I_{B}\right)$ is applied in two diagonals and the Hall voltage $\left(V_{H}\right)$ is measured between the remaining two corners (see Fig. $2 \mathrm{~b}$ ). To eliminate any offset voltage and magnetoresistance effects, it is important to change the direction of the current and magnetic field. The measurements for the Hall voltage are done in the following configurations, with south and north poles indicated by $S$ and $N$, respectively:

$$
\left.\begin{array}{clllll}
V_{H}^{24}: & I_{13}, & V_{24}{ }^{(S)} I_{13}, & V_{24}{ }^{(N)} I_{31}, & V_{42}{ }^{(S)} I_{31}, & V_{42}{ }^{(N)} \\
V_{H}^{13}: & I_{24}, & V_{13}{ }^{(S)} I_{24}, & V_{13}{ }^{(N)} I_{42}, & V_{31}{ }^{(S)} I_{42}, & V_{31}{ }^{(N)}
\end{array}\right\}
$$

This results in the following Hall voltage:

$$
V_{H}=\frac{\left(V_{24}^{S}-V_{24}^{N}\right)+\left(V_{42}^{S}-V_{42}^{N}\right)+\left(V_{13}^{S}-V_{13}^{N}\right)+\left(V_{31}^{S}-V_{31}^{N}\right)}{8}
$$

Finally, the carrier concentration $(n)$ and mobility $(\mu)$ can be calculated using the following equations from the Hall coefficient $R_{H}$ :

$$
R_{H}=\frac{V_{H}}{I_{B} B}=\frac{1}{e n}
$$

and

$$
\mu=\frac{R_{H}}{R_{s}}
$$

Van der Pauw instrumentation. The house-built measurement setup is capable of performing magneto-transport measurements in both ambient and vacuum, therefore providing a tool for both quick and more in-depth measurements. Figure $2 c$ shows the experimental setup: the sample is placed on a ceramic stage with a Pt100 heater attached underneath and electrical connections are made on top, through gold-plated contacts, featuring a half sphere contact and adjustable springs. It is important to ensure that the contacts are positioned right at the edges of the sample, as this can lead to errors in the measurement ${ }^{43}$. 


\begin{tabular}{|l|l|l|l|l|l|l|}
\hline \multicolumn{3}{|l|}{$R_{S}$ without field } & \multicolumn{3}{l|}{$V_{H}$ with field } \\
\hline & $R_{A}^{1,2}$ & $R_{A}^{3,4}$ & $R_{B}^{2,3}$ & $R_{B}^{4,1}$ & $V_{H}^{2,4}$ & $V_{H}^{1,3}$ \\
\hline $\boldsymbol{I}_{A C}^{+}$ & 4 & 2 & 1 & 3 & 1 & 4 \\
\hline $\boldsymbol{I}_{A C}^{-}$ & 3 & 1 & 4 & 2 & 3 & 2 \\
\hline$V_{A C}^{+}$ & 1 & 3 & 2 & 4 & 2 & 1 \\
\hline$V_{A C}^{-}$ & 2 & 4 & 3 & 1 & 4 & 3 \\
\hline
\end{tabular}

Table 1. Electrical combinations for measuring sheet resistance and Hall voltage in the van der Pauw geometry in AC bias-DC magnetic field configuration. For each resistance and Hall voltage measurement, the positive and negative terminals for the current bias and the voltage measurement are connected to the 4 contacts at the corners of the sample according to this configuration (Fig. 2a,b).

To measure the magneto-transport properties in vdP geometry, an Arduino microcontroller is used to trigger solid state relays, allowing current to pass and voltage measurements to be done at the different configurations as shown in Table 1 . Initially, the sheet resistance $\left(R_{s}^{v d P}\right)$ is calculated without magnetic field by applying an AC bias $\left(I_{B}=100 \mu A, f_{\text {current }} \approx 96 \mathrm{~Hz}\right)$ and measuring the voltage drop across the different configurations. The Hall effect is induced by an electromagnet coil that produced a DC magnetic field (15 $\mathrm{mT})$. The resulting Hall voltage $\left(V_{H}\right)$ response of the AC biased sample is measured. In both $R_{S}$ and $V_{H}$ measurements, the voltages are measured using a lock-in amplifier (LIA) referenced to the first harmonic of $f_{\text {current }}$, a technique which offers two advantages over DC: 1) eliminates the need for additional configurations and therefore reduces complexity; 2) allows minimum electrical noise in the system. The carrier concentration and mobility of the sample are calculated by using Eqs. (7) and (8).

THz-TDS map weighting for comparison with vdP measurements. The sheet resistance values $R_{s}^{T H z}$ obtained with TDS (see Section 2.3) and $R_{s}^{v d P}$ obtained with the vdP method (see Section 2.4.1) can be compared to establish a correlation between these two different but complementary methods. For a given sample, the vdP measured value $R_{s}^{v d P}$ is a weighted average,

$$
R_{s}^{v d P}=\frac{1}{A} \int_{A} w(P) \rho(P) \mathrm{d} A
$$

where $\rho(P)$ is the local sheet resistivity at point $P, \mathrm{~d} A$ is the surface element, and $w(P)$ is the normalized sensitivity function of the vdP method. Much work has been devoted by Koon and others ${ }^{44-47}$ to identify the general form of $w(P)$, which depends on the (strongly non-uniform) local current distribution associated with the vdP measurement, and to the $\rho(\mathrm{P})$ itself. The zero-order approximation for $w(P)$ is $w_{0}(P)$, the sensitivity function for a sample of uniform resistivity. The $w_{0}(P)$ was computed by numerical methods, for the case of a square sample with electrodes on its corners. For this case $w_{0}(P)$ has a bell shape (see Fig. $2 \mathrm{a}$ in ref. ${ }^{47}$ ), with a value of about 3.4 in the centre and vanishing on the square edges. Therefore, the resistivity near the centre affects $R_{s}^{v d P}$ more than that close to the sample border.

$R_{s}^{T H z}(P)$ has a constant sensitivity over $P$ being measured with a scanning technique, and as a result, the average $R_{s}^{T H z}$ is a non-weighted quantity. However, to perform a more accurate comparison we can also compute the weighted average $R_{s, w}^{T H z}$ of the TDS map values using the sensitivity function of the vdP measurement:

$$
R_{s, w}^{T H z}=\frac{1}{A} \int_{A} w_{0}(P) R_{s}^{T H z}(P) \mathrm{d} A
$$

\section{Results and Discussion}

In the following, we present and discuss results obtained on a batch of 6 samples of CVD graphene transferred on a single quartz substrate and diced into chips.

Optical inspection. Figure 3 shows a confocal laser scanning microscopy image of a $700 \times 700 \mu \mathrm{m}$ area within the centre of the sample. Representative images were taken from all the samples and for different areas to ensure the continued coverage of graphene. The zoom-in image of Fig. $3 \mathrm{~b}$ reveals details of the sample structure. It is apparent that the samples include 2-3LG islands (brighter spots). Moreover, the samples have several wrinkles due to the growth and transfer process (i.e. different thermal expansion coefficients of $\mathrm{Cu}$ and graphene). However, the continuity of the sample is maintained, and these features are not expected to affect the vdP or THz-TDS measurements.

Local work function measurements. When a single-atomic layer such as graphene is measured, surface contamination may lead to incorrect results. When graphene is transferred on a target substrate, the top surface is covered with polymer residues. In our measurements, the residues are removed prior to the measurement using contact-mode atomic force microscopy (AFM) to expose a clean surface. The results in ambient are shown in Fig. 4a,b, where the exposed cleaned area is highlighted with white dashed lines. Following calibration of the tip (Fig. 1), the work function map was constructed (Fig. 4b), demonstrating a work function difference between the dirty and cleaned area of $\sim 100 \mathrm{meV}$. This demonstrates the importance of cleaning the surface prior any 
(a)

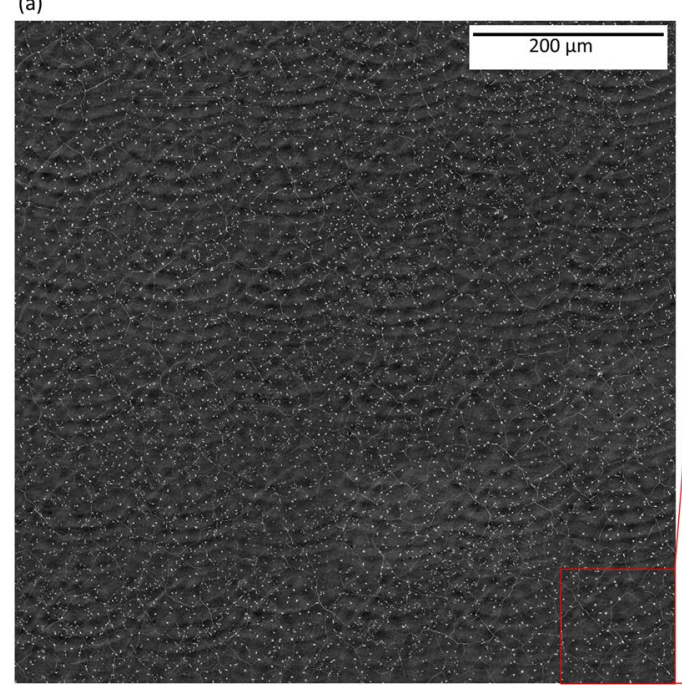

(b)

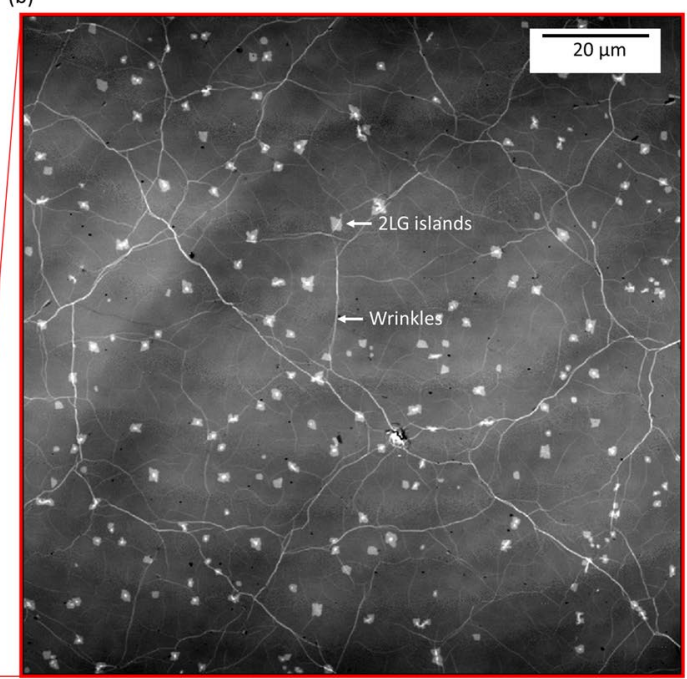

Figure 3. (a) Typical confocal laser scanning microscope optical image (pseudo colour) of a typical graphene sample (the fringe-like structures are artefacts of the method due to lens imperfections). (b) Zoom in area indicating 2-3LG islands and graphene wrinkles.

measurements. Moreover, 2-3LG islands demonstrate lower work functions than 1LG, due to screening of the substrate charges ${ }^{24,25}$. The summarized results of work function measurements of the different samples are shown in Fig. 4c, with the average work functions for $1 \mathrm{LG}, 2 \mathrm{LG}$ and $3 \mathrm{LG}$ in ambient conditions being $\Phi_{1 L G}=4.83 \mathrm{eV}$, $\Phi_{2 L G}=4.55 \mathrm{eV}$ and $\Phi_{3 L G}=4.35 \mathrm{eV}$, respectively.

THz-TDS measurements. Figure 5 shows the maps of the local sheet resistance $R_{s}^{T H z}(P)$ obtained using the Onyx THz-TDS system. From the maps it is evident that the sheet resistance features some spatial inhomogeneities, which highlights the need for a weighted average in order to compare the values to the vdP method. Average conductance and resistance values extracted from the $\mathrm{THz}$ maps and their standard deviations are summarised in Table 2.

The features observed in these maps have been reported in the literature in ${ }^{16,48}$, and they can be related to the presence of microscopic defects, such as cracks, and to the presence of growth domain. The measurements are affected by edge effects that span about $1 \mathrm{~mm}$ from the edge due to the size of the THz beam, which is estimated to be $0.5 \mathrm{~mm}$ in diameter. Most of the features show up as spots or islands of significantly higher sheet resistance, indicating a low quality of the graphene deposited in that particular area (e.g. samples 15, 22, 34 and 45). Other features show up as two distinct sheet resistance areas separated by a varying size transition zone (e.g. samples 3 and 39).

Van der Pauw measurements. Following the THz-TDS sheet resistance mapping, the magneto-transport properties of six samples were measured using the home-build vdP setup described in Section 2.4. VdP can be considered as a complementary method to THz-TDS measurements as the later one only provided information about the sheet resistance/conductance and can only be performed in ambient conditions. As both carrier concentration and mobility are essential parameters for the material performance, their measurements provide crucial information about the quality of the graphene layer ${ }^{25}$. Additional advantage of the vdP method is that using the current setup both ambient and vacuum measurements can be performed and thus the effect of the environmental exposure on the electrical properties of graphene can be studied.

The results of vdP measurements are shown in Fig. 6. Initially the samples were stored in a desiccator and then they were measured in ambient (relative humidity and temperature of $\sim 33 \%$ and $\sim 25^{\circ} \mathrm{C}$, respectively). The samples show a p-type behaviour with an average carrier concentration of $n_{h} \approx 1.7 \times 10^{13} \mathrm{~cm}^{-2}$ in ambient (Fig. 6a), with the error bar representing variations from individual measurements because of the time the samples needed to stabilize their properties. The average carrier mobility and sheet resistance are $\mu_{h} \approx 1000 \mathrm{~cm}^{2} \mathrm{~V}^{-1} \mathrm{~s}^{-1}$ and $R_{s}^{v d P} \approx 400 \Omega \mathrm{sq}^{-1}$, respectively. The carrier concentration vs mobility measurements show linear dependence with a slope of $-7.65 \times 10^{-11} \mathrm{~cm}^{4} \mathrm{~V}^{-1} \mathrm{~s}^{-1}$ (Fig. 6d). Although these measurements are fast and ideal for quick electrical characterisation of graphene, environmental fluctuations in the ambient air will affect the measurement, resulting in large variations in the measured values ${ }^{25}$. Therefore, using the dedicated measurement setup, the magneto-transport measurements in the van der Pauw geometry were also performed in vacuum. Prior the measurement, the samples were annealed $\left(170^{\circ} \mathrm{C}\right)$ in vacuum $(P=0.005 \mathrm{~Pa})$ for 3 hours and then they were cool down to room temperature. This resulted in desorption of atmospheric contaminants responsible for the high doping observed in ambient conditions from the graphene surface. This is evident from the significant reduction (increase) of the carrier concentration (mobility) (Fig. 6a,b), such as in vacuum the average carrier concentration and mobility were $n_{h} \approx 3 \times 10^{12} \mathrm{~cm}^{-2}$ and $\mu_{h} \approx 1800 \mathrm{~cm}^{2} \mathrm{~V}^{-1} \mathrm{~s}^{-1}$, respectively. Importantly, the large experimen- 
(a) Topography-AFM

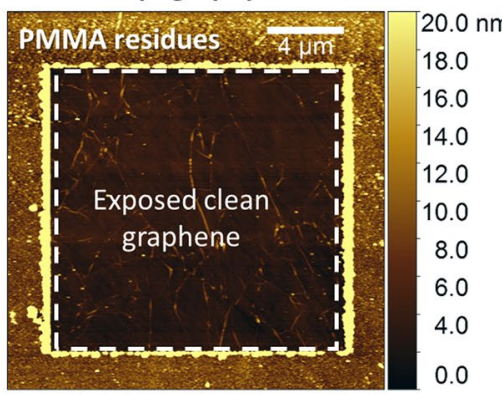

(b) Work function-KPFM

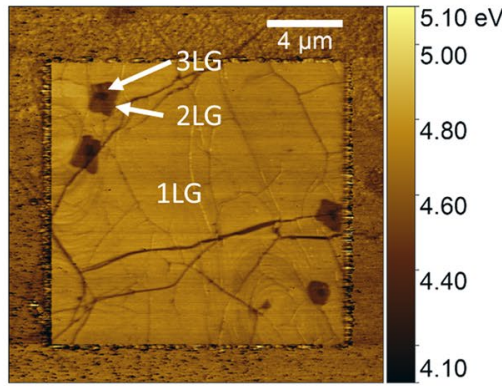

(c)

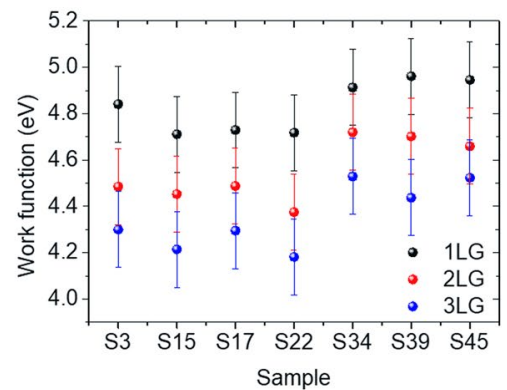

Figure 4. (a) Topography and (b) work function of a representative graphene sample on quartz substrate.

(c) Summary of work functions for the different graphene samples (black, red, blue dots for 1LG, 2LG and 3LG, respectively). The PMMA residues following contact mode cleaning were accumulated in a square around the cleaned area (indicated with dashed line in (a)).

(a)

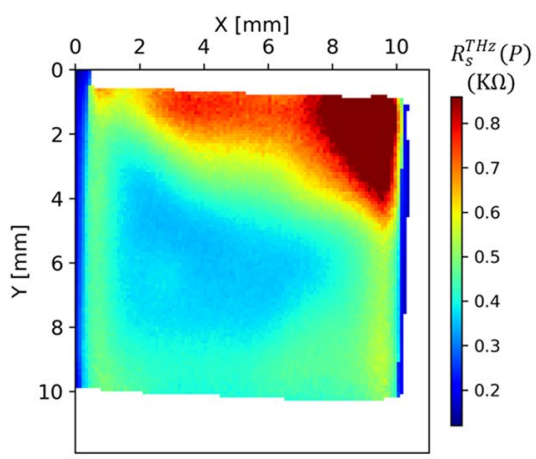

(d)

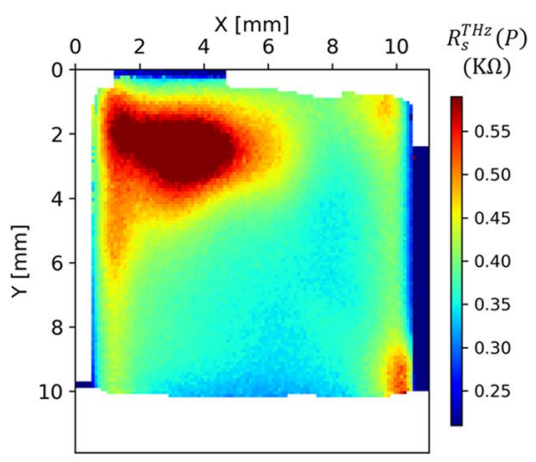

(b)

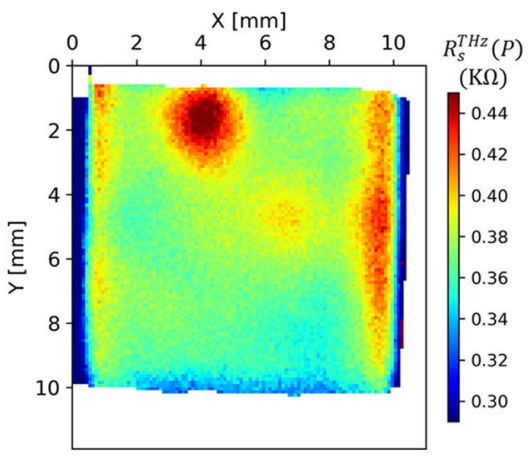

(e)

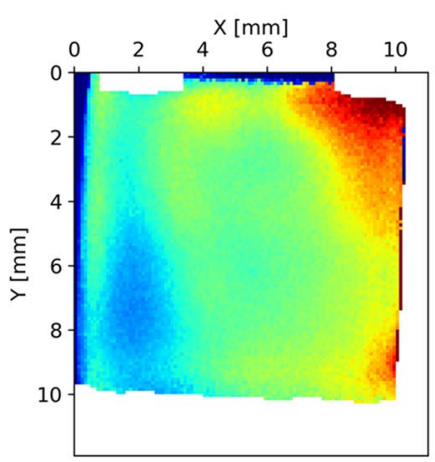

$R_{s}^{T H z}(P)$

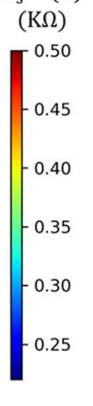

(c)

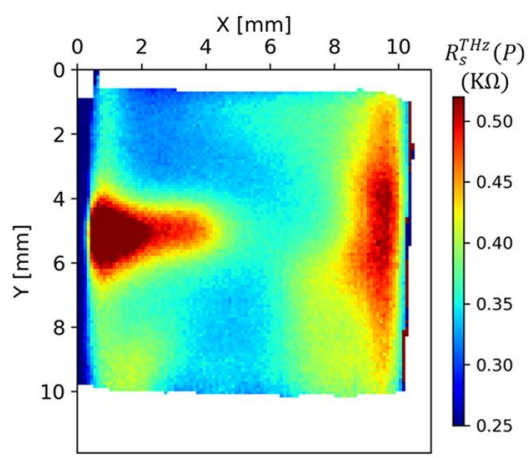

(f) Sample 45

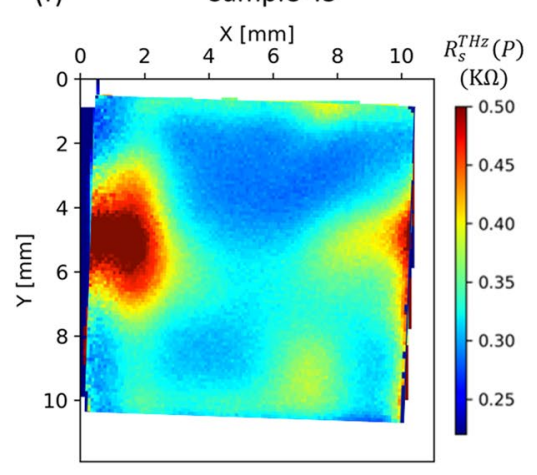

Figure 5. Sheet resistance maps of 6 samples obtained using the THz-TDS system, exposing areas of inhomogeneous sheet resistance.

\begin{tabular}{|l|l|l|l|}
\hline Sample & Average $(\mathbf{m S}) /(\mathbf{k} \boldsymbol{\Omega} / \mathbf{s q})$ & Standard deviation $(\mathbf{m S}) /(\mathbf{k} \boldsymbol{\Omega} / \mathbf{s q})$ & Variation coefficient $(\%)$ \\
\hline S3 & $2.16 / 0.46$ & $0.52 / 0.110$ & 24 \\
\hline S15 & $2.64 / 0.38$ & $0.13 / 0.019$ & 5 \\
\hline S22 & $2.65 / 0.37$ & $0.30 / 0.041$ & 11 \\
\hline S34 & $2.43 / 0.41$ & $0.37 / 0.062$ & 15 \\
\hline S39 & $2.82 / 0.35$ & $0.23 / 0.028$ & 8 \\
\hline S45 & $2.69 / 0.37$ & $0.33 / 0.052$ & 14 \\
\hline
\end{tabular}

Table 2. Average and standard deviation of the conductance/resistance measured from $\mathrm{THz}$ maps. Variation coefficient is the ratio between the standard deviation and the average. 
(a)

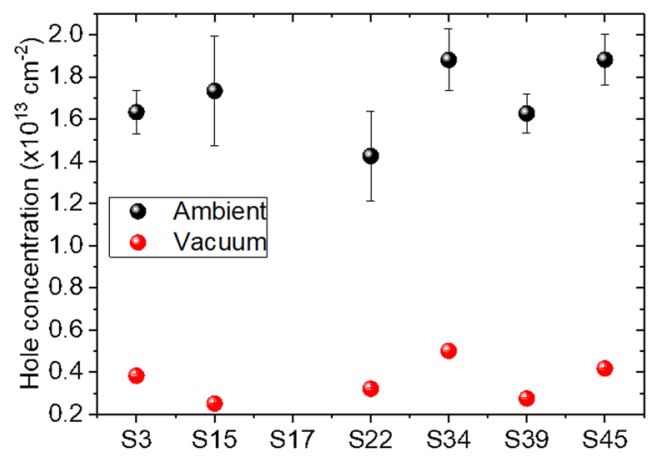

(c)

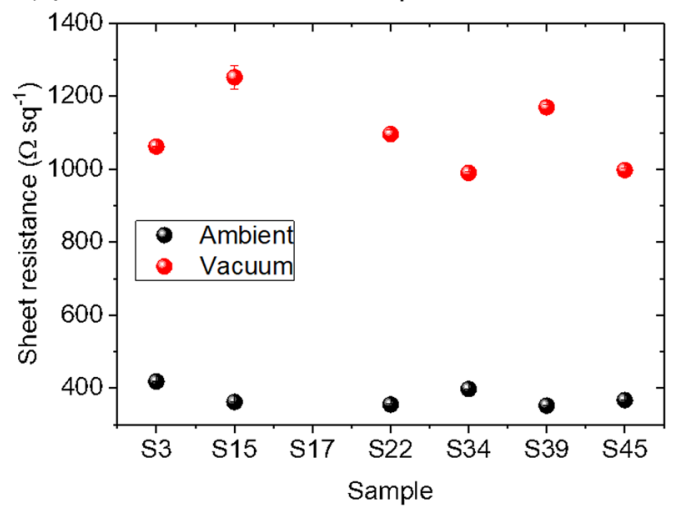

(b)
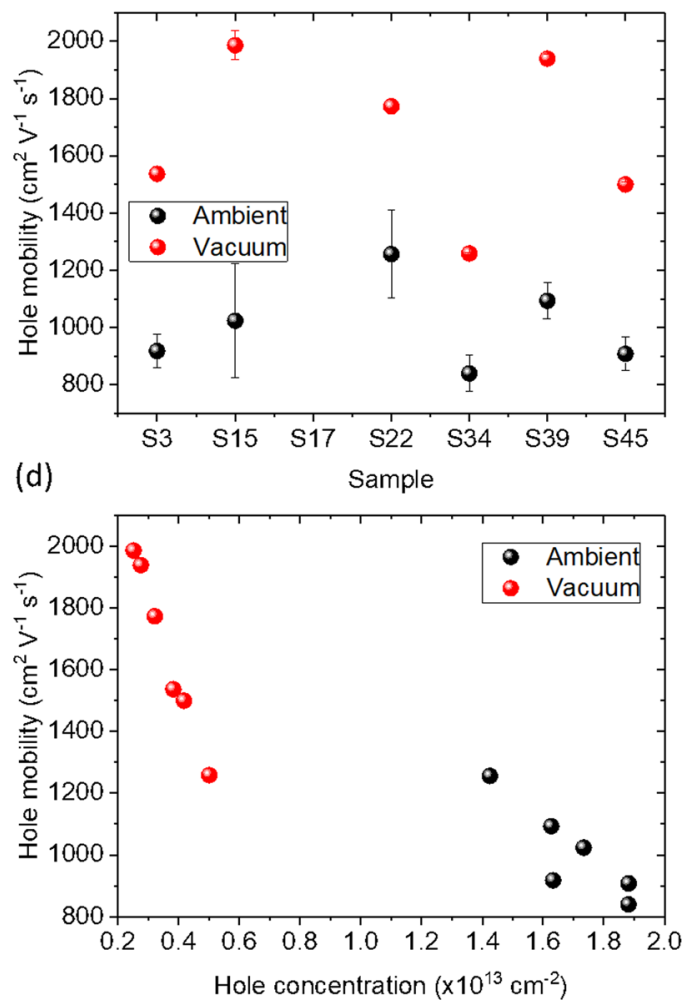

Figure 6. Magneto-transport measurements of the graphene samples in the van der Pauw geometry in ambient (black) and vacuum (red): (a) carrier concentration, (b) mobility, (c) sheet resistance for individual samples, (d) carrier concentration vs mobility dependence.

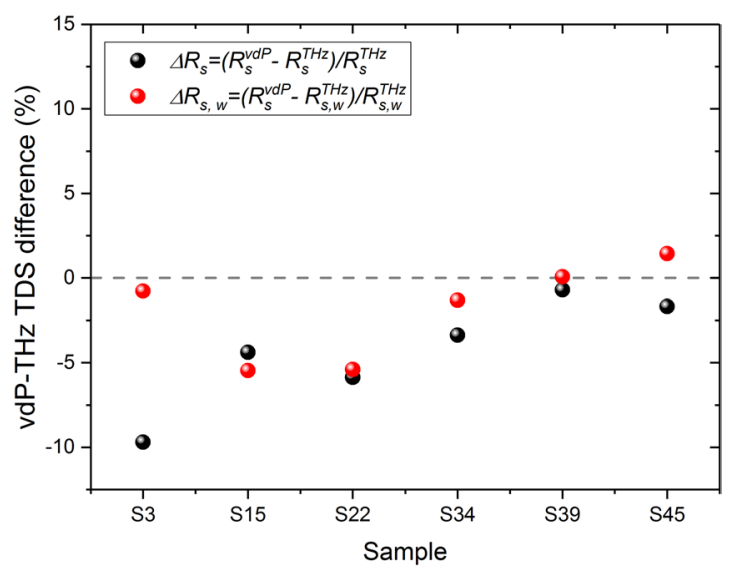

Figure 7. Comparison between the van der Pauw and THz-TDS methods. Black points represent the as measured difference $\Delta \boldsymbol{R}_{s}$, while red points represent the weighted difference $\Delta \boldsymbol{R}_{\boldsymbol{s}, \boldsymbol{w}}$.

tal error observed in ambient was significantly reduced in vacuum, allowing stable measurements. In vacuum, the sheet resistance of the samples increased to $R_{s}^{v d P} \approx 1300 \Omega \mathrm{sq}^{-1}$, due to the decreased number of carriers (Fig. 6c).

Comparison between vdP and THz-TDS characterisation. In order to compare the results of vdP and THz-TDS methods, the following equations were used to calculate the relative percent differences on resistance:

$$
\begin{gathered}
\Delta R_{s}=\left(R_{s}^{v d P}-R_{s}^{T H z}\right) / R_{s}^{T H z} \\
\Delta R_{s, w}=\left(R_{s}^{v d P}-R_{s, w}^{T H z}\right) / R_{s, w}^{T H z}
\end{gathered}
$$




\begin{tabular}{|c|c|c|c|}
\hline \multirow[b]{2}{*}{ Characteristics } & \multicolumn{3}{|l|}{ Techniques } \\
\hline & Van der Pauw & THz-TDS & KPFM \\
\hline Type & Contact & Contactless & Contactless \\
\hline Measured Quantity & $\begin{array}{l}\text { Carrier concentration, mobility, sheet } \\
\text { resistance/conductance }\end{array}$ & Sheet resistance/conductance & Work function \\
\hline Environmental Conditions & Ambient, vacuum, custom & Ambient, custom & Ambient, custom, vacuum \\
\hline Measurement Time & $\begin{array}{l}\sim 10 \text { seconds, though longer time for } \\
\text { conditioning of samples in vacuum } \\
\text { is needed }\end{array}$ & $\sim 1$ minute & $\begin{array}{l}\sim 30 \text { minutes, depending on scan size } \\
\text { and speed }\end{array}$ \\
\hline Manufacturing Integration & Off-line batch characterisation & Wafer-scale & Off-line sample characterisation \\
\hline $\begin{array}{l}\text { Measurement Scale } \\
\text { Spatial resolution }\end{array}$ & $\begin{array}{l}\text { Global (macro) } \\
\text { N/A }\end{array}$ & $\begin{array}{l}\text { Local (micro) } \\
1 \mathrm{~mm} \text { to } 50 \mu \mathrm{m}\end{array}$ & \begin{tabular}{l} 
Local (nano) \\
\hdashline 10 to $30 \mathrm{~nm}$
\end{tabular} \\
\hline Sources of error & $\begin{array}{l}\text { Non-ohmic contacts, discontinuities, } \\
\text { thermoelectric effects, ambient } \\
\text { conditions stability }\end{array}$ & $\begin{array}{l}\text { Roughness of the back side of } \\
\text { the sample }\end{array}$ & $\begin{array}{l}\text { Lack of grounding, contaminated } \\
\text { sample, wrong calibration }\end{array}$ \\
\hline
\end{tabular}

Table 3. Main characteristics of vdP, THz-TDS and KPFM measurement techniques.

where the quantities are defined by Eqs. 9-10. Although the series $\Delta R_{s}$ (black points in Fig. 7 following Eq. (12)) already demonstrates a good agreement between individual values, the series $\Delta R_{s, w}$ (red points following Eq. (13)) obtained after application of the vdP weighting function (Eqs. (9) and (10)) demonstrates that the residual difference between the two measurement methods is further reduced for the majority of the studied samples. For most of the samples, the relative differences are below $5 \%$, showing a very good quantitative agreement between $\mathrm{vdP}$ and THz-TDS methods.

A visual explanation of the correction can be given for sample $S 3$ as an example in Fig. 5 in which the conductivity is lower in the centre and higher around the border. Since the vdP measurement is more sensitive to the conductivity in the centre of the sample (see Section. 2.5), $R_{s}^{v d P}<R_{s}^{T H z}$. Therefore, in Fig. 7, $\Delta R_{s}<0$, and after applying the weighting corresponding to the vdP sensitivity function, the differences of sheet resistance measured between the two methods have been reduced.

\section{Conclusion}

The characteristics of each technique are summarised in Table 3, where it is evident that a variety of complimentary techniques is necessary to obtain a complete understanding of an unknown graphene sample. While the vdP method can provide an average value of the magneto-transport properties of the graphene sample (on all insulating substrates) in both ambient and vacuum conditions in seconds, it is a contact method and discontinuities can affect the measurement significantly. On the contrary, THz-TDS mapping is a contactless method, which provides information of the spatial variations of the sheet resistance of the graphene sample, but currently it does not support measurements of carrier concentration or mobility (without the need of modeling). Also, due to the scanning procedure, it inherently takes long time (several minutes) to complete a measurement. KPFM is currently the only available method, which can provide nanometer scale work function maps of graphene. For all the methods, care must be taken when a thin film of polymer residues covers the sample. Where appropriate, it should be taken into account that values of the electrical parameters corresponding to the intrinsic properties of graphene (as measured in vacuum) can vary significantly to those measured in ambient conditions due to the presence of airborne adsorbates.

In conclusion, we introduce a procedure for performing electrical measurements of CVD graphene from macro- to nano-scale using a variety of contact and contactless techniques. The sheet resistance, carrier concentration and mobility were measured using macroscale magneto-transport measurements in the vdP geometry, both in vacuum and ambient conditions to extract the pristine electrical properties of graphene. Upon annealing in vacuum, the carrier concentration has significantly decreased due to desorption of atmospheric molecules (such as water), while the carrier mobility and sheet resistance have significantly increased. The measurements were compared to the sheet resistance maps obtained using microscale THz-TDS mapping, where a weighted average function was applied. For most of the samples, the difference in the sheet resistance as obtained by vdP and THz-TDS was below 5\%, demonstrating the good agreement between the different methods. A method for calibrated KPFM measurements of graphene samples is demonstrated, allowing high resolution mapping of the work function on the nanoscale and highlighting the impact of polymer residues on the surface. This work highlights the importance of using a standardized methodology for electrical measurements using a variety of complimentary techniques to obtain a complete understanding of the sample on a variety of lateral scales. The development of these presented metrology and methodologies within the GRACE project ${ }^{41}$ will become an important part to contribute to the standards development work of the normative bodies, through the initiation of and dissemination of new written standards for the electrical characterisation of graphene based on the Good Practice Guides developed within the project.

\section{Methods}

Graphene growth. The mono-layer graphene growth was carried out in a cold-walled chemical vapour deposition (CVD) reactor (Aixtron BM) using Cu foil as the catalyst. Following the growth, PMMA was spin-coated onto the graphene-covered $\mathrm{Cu}$ foil, acting as a sacrificial support layer. The $\mathrm{Cu}$ foil was then etched using a $\mathrm{FeCl}_{3}$ containing solution. Distilled water was used to clean the film several times after it was transferred onto a quartz 
substrate. Finally, the PMMA layer was removed by immersion in acetone. In this work we use $4^{\prime \prime}$ CVD graphene sample, which was diced into $10 \times 10 \mathrm{~mm}^{2}$ squares. Several diced chips were chosen in random and studied by a number of methods as discussed.

\section{Data availability}

The datasets generated during and/or analysed during the current study are available from the corresponding author on reasonable request.

Received: 1 May 2019; Accepted: 5 December 2019;

Published online: 21 February 2020

\section{References}

1. Ferrari, A. C. et al. Science and technology roadmap for graphene, related two-dimensional crystals, and hybrid systems. Nanoscale 7(11), 4598-4810 (2014).

2. Li, X. et al. Large-area synthesis of high-quality and uniform graphene films on copper foils. Science 324(5932), 1312-1314 (2009).

3. Mattevi, C., Kim, H. \& Chhowalla, M. A review of chemical vapour deposition of graphene on copper. J. Mater. Chem. 21(10), 3324 (2011).

4. Rosei, R. et al. Structure of graphitic carbon on $\mathrm{Ni}(111)$ : A surface extended-energy-loss fine-structure study. Phys. Rev. B. 28(2), 1161-1164 (1983).

5. Shikin, A. M., Prudnikova, G. V., Adamchuk, V. K., Moresco, F. \& Rieder, K. H. Surface intercalation of gold underneath a graphite monolayer on $\mathrm{Ni}(111)$ studied by angle-resolved photoemission and high-resolution electron-energy-loss spectroscopy. Phys. Rev. B - Condens. Matter Mater. Phys. 62(19), 13202-13208 (2000).

6. Varykhalov, A. et al. Electronic and Magnetic Properties of Quasifreestanding Graphene on Ni. Phys. Rev. Lett. 101(15), 157601, (Oct. 2008).

7. Sutter, P. W., Flege, J.-I. \& Sutter, E. A. Epitaxial graphene on ruthenium. Nat. Mater. 7(5), 406-411 (May 2008).

8. Zi-Pu, H., Ogletree, D. F., Van Hove, M. A. \& Somorjai, G. A. Leed theory for incommensurate overlayers: Application to graphite on Pt(111). Surf. Sci. 180(2-3), 433-459 (1987).

9. Preobrajenski, A. B., Ng, M. L., Vinogradov, A. S. \& Mårtensson, N. Controlling graphene corrugation on lattice-mismatched substrates. Phys. Rev. B - Condens. Matter Mater. Phys. 78(7), 2-5 (2008).

10. Kholin, N. A., Rut'kov, E. V. \& Tontegode, A. Y. The nature of the adsorption bond between graphite islands and iridium surface. Surf. Sci. 139(1), 155-172 (1984).

11. Pletikosić, I. et al. Dirac Cones and Minigaps for Graphene on Ir(111). Phys. Rev. Lett. 102(5), 056808 (Feb. 2009).

12. Mortazavi Zanjani, S. M., Holt, M., Sadeghi, M. M., Rahimi, S. \& Akinwande, D. 3D integrated monolayer graphene-Si CMOS RF gas sensor platform. npj 2D Mater. Appl. 1(1), 36 (2017).

13. Han, S.-J., Garcia, A. V., Oida, S., Jenkins, K. A. \& Haensch, W. Graphene radio frequency receiver integrated circuit. Nat. Commun. 5, 1-6 (Jan. 2014).

14. Goossens, S. et al. Broadband image sensor array based on graphene-CMOS integration. Nat. Photonics 11(6), 366-371 (2017).

15. Petersen, D. H., Hansen, O., Lin, R. \& Nielsen, P. F. Micro-four-point probe Hall effect measurement method. J. Appl. Phys. 104(1) (2008).

16. Bøggild, P. et al. Mapping the electrical properties of large-area graphene, 2D Mater. 4(4), 042003 (Sep. 2017).

17. Hao, L. et al. Non-contact method for measurement of the microwave conductivity of graphene. Appl. Phys. Lett. 103(12), 123103 (2013).

18. Shaforost, O. et al. Contact-free sheet resistance determination of large area graphene layers by an open dielectric loaded microwave cavity. J. Appl. Phys. 117(2) (2015).

19. Cooke, D. G. et al. Transient terahertz conductivity in photoexcited silicon nanocrystal films. Phys. Rev. B - Condens. Matter Mater. Phys. 73(19), 1-4 (2006).

20. Frenzel, A. J. et al. Observation of suppressed terahertz absorption in photoexcited graphene. Appl. Phys. Lett. 102(11) (2013).

21. A. Cultrera, et al., Mapping the conductivity of graphene with Electrical Resistance Tomography, Scientific Reports, 9, 10655 (2019).

22. Ren, L. et al. Terahertz and infrared spectroscopy of gated large-area graphene. Nano Lett. 12(7), 3711-3715 (2012).

23. Buron, J. D. et al. Graphene Conductance Uniformity Mapping. Nano Lett. 12(10), 5074-5081 (Oct. 2012).

24. Melios, C. et al. Effects of humidity on the electronic properties of graphene prepared by chemical vapour deposition. Carbon N. Y. 103, 273-280 (2016).

25. Melios, C., Giusca, C. E., Panchal, V. \& Kazakova, O. Water on graphene: review of recent progress. 2D Mater. 5(2), 022001 (Jan. 2018).

26. Chwang, R., Smith, B. J. \& Crowell, C. R. Contact size effects on the van der Pauw method for resistivity and Hall coefficient measurement. Solid State Electron. 17(12), 1217-1227 (1974).

27. van der Pauw, L. J. A Method of Measuring the Resistivity and Hall Coefficient on Lamellae of Arbitrary Shape. Philips. Tech. Rev. 20(220-224) (1958).

28. van der Pauw, L. J. A Method of Measuring Specific Resistivity and Hall effect of Discs of Arbitrary Shape. Philips. Res. Reports 13(1), $1-11(1958)$.

29. Mackenzie, D. M. A. et al. Quality assessment of terahertz time-domain spectroscopy transmission and reflection modes for graphene conductivity mapping. Opt. Express (2018).

30. Buron, J. D. et al. Electrically continuous graphene from single crystal copper verified by terahertz conductance spectroscopy and micro four-point probe. Nano Lett. (2014).

31. Tomaino, J. L. et al. Terahertz imaging and spectroscopy of large-area single-layer graphene. Opt. Express (2011).

32. Hibino, H. et al. Dependence of electronic properties of epitaxial few-layer graphene on the number of layers investigated by photoelectron emission microscopy. Phys. Rev. B. 79(12), 1-7 (Mar. 2009).

33. Melitz, W., Shen, J., Kummel, A. C. \& Lee, S. Kelvin probe force microscopy and its application. Surf. Sci. Rep. 66(1), 1-27 (Jan. 2011).

34. Nonnenmacher, M., O’Boyle, M. P. \& Wickramasinghe, H. K. Kelvin probe force microscopy. Appl. Phys. Lett. 58(25), 2921-2923 (1991).

35. Panchal, V., Pearce, R., Yakimova, R., Tzalenchuk, A. \& Kazakova, O., Standardization of surface potential measurements of graphene domains. Sci. Rep. 3(2), 2597 (Sep. 2013).

36. Panchal, V. et al. Surface potential variations in epitaxial graphene devices investigated by Electrostatic Force Spectroscopy. In 2012 12th IEEE International Conference on Nanotechnology (IEEE-NANO), pp. 1-5 (2012).

37. Panchal, V., Giusca, C. E., Lartsev, A., Yakimova, R. \& Kazakova, O. Local electric field screening in bi-layer graphene devices. Front. Phys., 2(February, 27-31 (2014).

38. Kazakova, O., Panchal, V. \& Burnett, T. Epitaxial Graphene and Graphene-Based Devices Studied by Electrical Scanning Probe Microscopy. Crystals, 3(1), 191-233, (Mar 2013). 
39. Ziegler, D. et al. Variations in the work function of doped single- and few-layer graphene assessed by Kelvin probe force microscopy and density functional theory. Phys. Rev. B. 83(23), 1-7 (Jun. 2011).

40. Melios, C. et al. Carrier type inversion in quasi-free standing graphene: studies of local electronic and structural properties. Sci. Rep. 5, 10505 (2015).

41. 16NRM01 GRACE: Developing electrical characterisation methods for future graphene electronics" is a Joint Research Project of the European Metrology Programme for Innovation and Research (EMPIR). Project website http://empir.npl.co.uk/grace/

42. Panchal, V. et al. Confocal laser scanning microscopy for rapid optical characterization of graphene. Commun. Phys. 1(1), 83 (Dec. 2018).

43. Koon, D. W. Effect of contact size and placement, and of resistive inhomogeneities on van der Pauw measurements. Rev. Sci. Instrum. 60(2), 271-274 (Feb. 1989).

44. Koon, D. W. \& Knickerbocker, C. J. What do you measure when you measure resistivity?. Rev. Sci. Instrum. 63(1), 207-210 (Jan. 1992).

45. Koon, D. W. \& Chan, W. K. Direct measurement of the resistivity weighting function. Rev. Sci. Instrum. (1998).

46. Koon, D. W., Wang, F., Petersen, D. H. \& Hansen, O. Sensitivity of resistive and Hall measurements to local inhomogeneities: Finitefield, intensity, and area corrections. J. Appl. Phys. 116(13) (2014).

47. Koon, D. W., Heřmanová, M. \& Náhlík, J. Electrical conductance sensitivity functions for square and circular cloverleaf van der Pauw geometries. Meas. Sci. Technol. 26, 115004 (2015).

48. Whelan, P. R. et al. Electrical Homogeneity Mapping of Epitaxial Graphene on Silicon Carbide. ACS Appl. Mater. Interfaces 10, 31641 (2018).

\section{Acknowledgements}

The work has been developed within the Joint Resarch Project 16NRM01 GRACE: Developing electrical characterisation methods for future graphene electronics ${ }^{41}$. This project has received funding from the EMPIR programme co-financed by the Participating States and from the European Union's Horizon 2020 research and innovation programme. NPL authors acknowledge funding from the European Union's Horizon 2020 research and innovation programme under grant agreement GrapheneCore2 785219 number.

\section{Author contributions}

L.C. and O.K. proposed the idea of manuscript. Alba Centeno, O.T. and A.Z. fabricated the samples. C.M., N.H., Alessandro Cultrera, Alvaro Cordon, V.P., I.A., A.R.-S., D.E., M.F., A.L. and S.R. performed the measurements. All authors participated in writing and reviewed the manuscript.

\section{Competing interests}

The authors declare no competing interests.

\section{Additional information}

Supplementary information is available for this paper at https://doi.org/10.1038/s41598-020-59851-1.

Correspondence and requests for materials should be addressed to O.K.

Reprints and permissions information is available at www.nature.com/reprints.

Publisher's note Springer Nature remains neutral with regard to jurisdictional claims in published maps and institutional affiliations.

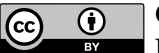

Open Access This article is licensed under a Creative Commons Attribution 4.0 International License, which permits use, sharing, adaptation, distribution and reproduction in any medium or format, as long as you give appropriate credit to the original author(s) and the source, provide a link to the Creative Commons license, and indicate if changes were made. The images or other third party material in this article are included in the article's Creative Commons license, unless indicated otherwise in a credit line to the material. If material is not included in the article's Creative Commons license and your intended use is not permitted by statutory regulation or exceeds the permitted use, you will need to obtain permission directly from the copyright holder. To view a copy of this license, visit http://creativecommons.org/licenses/by/4.0/.

(c) The Author(s) 2020 